\title{
Neoadjuvant chemotherapy with cisplatin, 5-FU, and leucovorin (PLF) in locally advanced gastric cancer: a prospective phase II study
}

\author{
Katja OtT $^{1}$, Andreas Sendler ${ }^{1}$, Karen Becker $^{2}$, Hans-Joachim Dittler ${ }^{1}$, Hermann Helmberger ${ }^{3}$, \\ Raimonde Busch $^{4}$, Christian Kollmannsberger ${ }^{1}$, J. Rüdiger Siewert ${ }^{1}$, and Ulrich Fink ${ }^{1}$ \\ ${ }^{1}$ Department of Surgery, Klinikum rechts der Isar der Technischen Universität München, Ismaninger Str. 22, 81675 München, Germany \\ ${ }^{2}$ Institute of Pathology, Technische Universität München, München, Germany \\ ${ }^{3}$ Department of Diagnostic Radiology, Klinikum rechts der Isar der Technischen Universität München, München, Germany \\ ${ }^{4}$ Institute of Medical Statistics and Epidemiology, Technische Universität München, München, Germany
}

\begin{abstract}
Background. Patients with locally advanced gastric cancer (cT3, cT4, N+, M0) have a dismal prognosis, despite complete resection. The objective of this study was to evaluate the toxicity and efficacy of neoadjuvant chemotherapy using the PLF (cisplatin/leucovorin [folinic acid]/5-fluorouracil [FU]) regimen in these patients. Primary endpoints of the study were the toxicity and the response to chemotherapy. Secondary endpoints were the rate of complete resection, survival, and first site of failure.

Methods. Forty-nine patients with adenocarcinoma of the stomach were enrolled. Staging was based on abdominal computed tomography (CT) scans, endosonography, and laparoscopy. The intention was to administer two cycles (each containing six courses) of preoperative chemotherapy, consisting of cisplatin $50 \mathrm{mg} / \mathrm{m}^{2}$, high-dose folinic acid (HD-FA) $500 \mathrm{mg} / \mathrm{m}^{2}$, and HD 5-FU (HD-5-FU) $2000 \mathrm{mg} / \mathrm{m}^{2}$ (PLF). Following chemotherapy all patients were referred to surgery. To be evaluable for response, survival, and first site of failure, the patient had to receive at least one cycle of chemotherapy.

Results. Toxicity observed was low, with grade 3 toxicity in fewer than $5 \%$ of the patients and two events of grade 4 toxicity (diarrhea and pulmonary embolism). Forty-two of the patients $(86 \%)$ received at least one cycle of chemotherapy. The clinical response rate in these patients was $26 \%$ (11/42 patients). In $76 \%$ of the patients (32/42), a complete resection was possible. The median duration of follow-up for the surviving patients was 58 months (range, 38 to $80+$ months). The median survival time for the 42 patients assessable for response was 25.4 months (range, 6 to $80+$ months). After complete resection, median survival time was 32 months (range, 7.6 to $80+$ months). The median survival time for clinically responding patients has not yet been determined, but 5-year survival is $\mathbf{9 0} \%$. Twenty of the 32 completely resected patients $(62.5 \%)$ had recurrences. First site of failure was peritoneal dissemination in 10 patients; locoregional and distant recurrences were rare.
\end{abstract}

Offprint requests to: A. Sendler

Received: March 17, 2003 / Accepted: May 27, 2003
Conclusion. Neoadjuvant chemotherapy with PLF in patients with locally advanced gastric cancer has low toxicity and reasonable efficacy, allowing administration on an outpatient basis. Clinically responding patients have an excellent outcome after complete resection. The development of peritoneal dissemination even after neoadjuvant chemotherapy and complete resection remains an unsolved problem in patients with nonintestinal type tumors.

Key words Stomach · Adenocarcinoma · Neoadjuvant chemotherapy

\section{Introduction}

Two-thirds of patients with gastric cancer in Western countries present with locally advanced disease (cT3-4, $\mathrm{Nx}, \mathrm{M} 0$ ). Even after complete resection, the 5-year survival in these patients is only about $25 \%$ [1,2]. In order to improve the relapse-free and overall survival in these patients, neoadjuvant chemotherapy has gained increasing interest in recent years [3-5]. Several phase II studies reported promising results with neoadjuvant chemotherapy in patients with potentially resectable [6-8] and locally advanced tumors [9-12]. The term "locally advanced gastric cancer" indicates that a complete resection (International Union Against Cancer [UICC]-R0) may be achieved in only about $40 \%$ of the patients [13].

Meticulous staging is a prerequisite for any kind of neoadjuvant chemotherapy. In this study, staging was based on endoscopy, endoluminal ultrasound (EUS), and computed tomography (CT) scan of the abdomen. To detect peritoneal dissemination, laparoscopy was performed in all patients. Studies of neoadjuvant chemotherapy in gastric cancer have so far demonstrated that the therapy is feasible and does not lead to an increase in postoperative morbidity and mortality. However, conclusive phase III studies are lacking. We, 
here, report the results of a single-center, prospective phase II study of neoadjuvant chemotherapy in locally advanced gastric cancer.

Combination chemotherapy, such as 5-fluorouracil (FU)/adriamycin/methotrexate (FAMTX) or etoposide/adriamycin/cisplatin (EAP) used in the treatment of gastric cancer achieved response rates of $20 \%-$ $25 \%$; however, toxicities were substantial, requiring hospital-based chemotherapy $[14,15]$. Therefore, there is a need for an effective but less toxic neoadjuvant treatment regimen, which can be administered in an outpatient fashion. In this respect, we investigated the PLF regimen (cisplatin/leucovorin [folinic acid]/5-FU). Primary endpoints of the study were toxicity and clinical response. Secondary endpoints were the rate of complete resection, survival, and first site of failure.

\section{Patients and methods}

\section{Patient selection and staging}

Between January 1995 and June 1997, 49 patients with previously untreated, biopsy-proven, locally advanced adenocarcinoma of the proximal and distal stomach were eligible for the study. The patients' characteristics are summarized in Table 1 . The study was approved by the local ethics committee and all patients gave written

Table 1. Patients' characteristics

\begin{tabular}{lll}
\hline Characteristic & \multicolumn{2}{l}{ No. of patients } \\
\hline Total & $49^{\mathrm{a}}$ & $42^{\mathrm{b}}$ \\
$\quad$ Men & 34 & 30 \\
$\quad$ Women & 15 & 12 \\
Age (years) & 58 & 56 \\
$\quad$ Median & $27-72$ & $27-72$ \\
$\quad$ Range & & \\
Performance status (WHO) & 0 & 0 \\
$\quad$ Median & $0-1$ & $0-1$ \\
$\quad$ Range & & \\
Location of primary tumor & 26 & 21 \\
$\quad$ Proximal & 23 & 21 \\
$\quad$ Middle, distal & & \\
Grading & 41 & 37 \\
$\quad$ Poorly differentiated & 8 & 5 \\
$\quad$ Moderately differentiated & & \\
T Category (EUS) & 38 & 32 \\
$\quad$ uT3 & 11 & 10 \\
$\quad$ LT4 & & \\
$\quad$ Intestinal type \\
$\quad$ Nonintestinal type \\
$\quad$ (diffuse and mixed)
\end{tabular}

EUS, endoluminal ultrasound

${ }^{a}$ Patients enrolled in the study

${ }^{\mathrm{b}}$ Patients evaluable informed consent. Inclusion criteria included the following: tumor category, cT3,4, Nx, M0; fit for surgery; age 18-70 years; not pregnant. Participating patients were, furthermore, required to have a WHO performance status of 0 or 1 ; adequate bone marrow function (WBC, $>4 \times 10^{9} /$; absolute neutrophil count [ANC], $>2 \times 10^{9} / 1$; platelets, $\left.>100 \times 10^{9} / 1\right)$; adequate renal function (creatinine clearance, $>60 \mathrm{ml} / \mathrm{min}$ and serum creatinine, $<1.5 \mathrm{mg} / \mathrm{dl}$ ); and adequate liver function (bilirubin level, $<1.5 \mathrm{mg} / \mathrm{dl}$ ). Exclusion criteria were: tumor category cT1-2; distant spread (M1), including proven peritoneal carcinomatosis; episodes of severe tumor bleeding; clinically evident severe gastric outlet syndrome; WHO performance status more than 1; medically unfit for surgery; age over 70 years; prior surgery to the stomach; secondary malignancies; and prior radio- or chemotherapy.

Pretreatment assessment included complete blood cell count with differential and serum multichannel chemical analysis. Staging to confirm consisted of upper gastrointestinal (GI) endoscopy with EUS. Diagnostic laparoscopy was obligatory for all patients. A CT scan of the entire abdomen was done with Gastrografine (Schering, Berlin, Germany) as a positive contrast and paraffin as a negative contrast in hypotonia. All patients had a chest radiogram.

Prior to the patients' inclusion in the study, a surgical oncologist, a medical oncologist, and a radiologist jointly evaluated all patients to determine whether the primary tumor was "locally advanced" and whether the patient was medically fit to undergo subsequent major surgery. All eligible patients had the choice of proceeding with an immediate operation if they did not wish to participate in this study.

\section{Chemotherapy}

Prior to chemotherapy an intravenous port system was implanted in all patients. The patients were treated with the PLF regimen, consisting of cisplatin $50 \mathrm{mg} / \mathrm{m}^{2}$, highdose folinic acid (HD-FA) $500 \mathrm{mg} / \mathrm{m}^{2}$, and HD 5-FU (HD-5-FU) $2000 \mathrm{mg} / \mathrm{m}^{2}$ [16]. Cisplatin was given on days 1,15 , and 29 as a 1-h infusion; HD-FA was administered on days $1,8,15,22,29$, and 36 as a 2 -h infusion, followed by HD-5-FU as a continuous infusion via a portable pump and an intravenous port system over $24 \mathrm{~h}$. Before the administration of cisplatin, the patients routinely received intravenous hydration and antiemetic therapy, including 5-HT3 antagonists and dexamethasone. Two cycles (each consisting of six courses of chemotherapy) were planned for each patient. The second cycle was scheduled following a 2-week rest after the first cycle.

Complete blood cell counts and serum chemistry (including levels of serum electrolytes and serum creati- 
nine) were performed at least once a week. The toxicity of the chemotherapy was graded according to WHO criteria. If there was anemia of more than grade 2, leukopenia of more than grade 3 , thrombocytopenia of more than grade 2; diarrhea, of more than grade 2, or mucositis of more than grade 2 on the day at administration, treatment was delayed until leukocyte counts and/or platelet counts had reached $1.5 \times 10^{\%} / 1$ or $1.0 \times 10^{9} / 1$, or until there was full recovery of all nonhematologic side effects, respectively. In regard to grade 3 nonhematologic toxicity and/or grade 3 (at the nadir) hematologic toxicity, the doses of 5-FU and cisplatin were decreased by $20 \%$ on all following treatment courses. After the second cycle, a resection was performed in all patients. If there was intolerable toxicity, deterioration of the clinical performance status, or tumor progression, chemotherapy was discontinued and the patients were referred to surgery. No subsequent postoperative chemo- or radiotherapy was scheduled after resection.

\section{Response evaluation}

To be assessable for response, patients had to receive at least one complete cycle of chemotherapy. Endoscopy with EUS and a CT scan of the abdomen were performed after every cycle of chemotherapy. Patients with clinically complete response (cCR) or clinically partial response (cPR), i.e., more than $50 \%$ decrease of tumor size on EUS and CT scan and total flattening of the gastric wall on endoscopy, were considered "responders" $[17,18]$. A cCR was defined as the complete disappearance of all lesions. Patients with minor responses (MR; less than $50 \%$ decrease of tumor size), no change situation (NC), and tumor progression (PD) were categorized as "non-responders". If progression of the disease was evident on one imaging technique only, it was scored as progression.

\section{Surgery}

Resection was performed after 2 weeks' rest following the last administration of chemotherapy. The standards for resection in the department were recently published elsewhere [13]. In most patients a total gastrectomy was performed. Transhiatal extended gastrectomy was done in tumors of the proximal third of the stomach or the anatomical cardia. In two patients, the tumor extended from the cardia to the distal esophagus. In these patients transhiatal esophagectomy and proximal gastrectomy was performed. An extended D2-lymphadenectomy, according to the rules of the Japanese Research Society for Gastric Cancer [19], was done in all patients.

\section{Surgical pathology, follow-up, and statistical analysis}

The $\mathrm{pT}, \mathrm{pN}, \mathrm{M}$, and $\mathrm{R}$ categories were assessed according to the guidelines of the UICC/American Joint Committee on Cancer (AJCC) [20,21]. The pathohistological workup was strictly standardized. The whole primary tumor was embedded in paraffin and sectioned in 5-mm slices. At least 30 tissue blocks for each tumor were stained with H\&E and investigated. To reveal even small subsets of tumor cells, an additional immunohistochemistry analysis, using an antibody against cytokeratin (CK 1/3; Dako Diagnostics, Hamburg, Germany) was done. A major pathological response was determined if fewer than $10 \%$ of vital tumor cells were found in the whole resected specimen [22].

Follow-up assessment consisted of a complete physical examination, endoscopy, chest X-ray, abdominal ultrasound, and an abdominal CT scan. Patients were assessed every 3 months during the first and second year and every 6 months thereafter.

All survival data were calculated from the date of first chemotherapy to date of death or the most recent follow-up. The survival rate was determined by the Kaplan-Meier method. Differences between the survival curves were calculated with the log rank test. All survival analyses were carried out using binary coded variables, and all test decisions were performed at a significance level of $P<0.05$.

\section{Results}

Preoperative chemotherapy, toxic effects, and clinical response

A total of 494 courses of chemotherapy were delivered to 49 patients. In 7 patients $(14 \%)$, chemotherapy was stopped during the first cycle. One patient developed a severe pulmonary embolism, and in 2 patients a subclavian vein thrombosis occurred. One patient developed grade 3 diarrhea and refused further chemotherapy. Two other patients were taken off study due to deterioration of their overall performance status. One patient demonstrated tumor progression during the first cycle. Following that, $42(86 \%)$ of the 49 patients enrolled in the study were finally evaluable for response, survival, and first site of failure. Four patients completed only one cycle of chemotherapy. This was due to a significant reduction of the performance status in 2 patients, a "no-change" situation in 1 patient, and a progression of the primary in 1 patient. The remaining 38 patients ( $77.5 \%$ of all patients) received the scheduled two cycles.

Toxic side effects during chemotherapy are summarized in Table 2. Hematological toxicity was low, with only three patients developing leucopenia grade 3 . There was one hospitalization due to chemotherapy- 
Table 2. Occurrence of hematological and nonhematological chemotherapy-induced toxic effects during neoadjuvant PLF treatment in patients with locally advanced gastric cancer $(n=49)$

\begin{tabular}{|c|c|c|c|c|c|}
\hline \multirow[b]{2}{*}{ WHO Grade } & \multicolumn{5}{|c|}{ Number of events (\%) } \\
\hline & 0 & 1 & 2 & 3 & 4 \\
\hline \multicolumn{6}{|l|}{ Toxic effect } \\
\hline Anemia & $6(12)$ & $28(57)$ & $13(27)$ & $2(4)$ & 0 \\
\hline Leukopenia & $15(31)$ & $11(22)$ & $20(41)$ & $3(6)$ & 0 \\
\hline Thrombocytopenia & $43(88)$ & $3(6)$ & $3(6)$ & 0 & 0 \\
\hline Emesis & $33(67)$ & $11(23)$ & $4(8)$ & $1(2)$ & 0 \\
\hline Nausea & $8(16)$ & $29(60)$ & $9(18)$ & $3(6)$ & 0 \\
\hline Mucositis & $30(61)$ & $19(39)$ & 0 & 0 & 0 \\
\hline Diarrhea & $35(72)$ & $8(16)$ & $2(4)$ & $3(6)$ & $1(2)$ \\
\hline Epistaxis & $32(65)$ & $12(25)$ & $5(10)$ & 0 & 0 \\
\hline Cutaneous & $45(92)$ & $4(8)$ & 0 & 0 & 0 \\
\hline Alopecia & $41(84)$ & 7 (14) & $1(2)$ & 0 & 0 \\
\hline $\begin{array}{l}\text { Thrombosis/ } \\
\text { Embolism (CTC) }\end{array}$ & $42(86)$ & 0 & 0 & $6(12)$ & $1(2)$ \\
\hline
\end{tabular}

PLF; cisplatin, $50 \mathrm{mg} / \mathrm{m}^{2}$; high-dose folinic acid (HD-FA), $500 \mathrm{mg} / \mathrm{m}^{2}$; and HD 5-fluorouracil (HD-5-FU), $2000 \mathrm{mg} / \mathrm{m}^{2}$

CTC, Common Toxicity Criteria Version 2.0, National Cancer Institute, 1999

induced toxicity (leucopenia grade 3 together with diarrhea grade 4). Most severe toxic complications were due to thrombosis of the subclavian vein at the site of the implanted port system (six patients), and pulmonary embolism in one patient. Of these seven patients, three patients were taken off study during the first cycle.

Clinical response evaluation demonstrated a partial response in $11(26 \%)$ patients, minor responses in 20 $(48 \%)$, "no change" in $8(19 \%)$, and locoregional progressive disease in $3(7 \%)$ patients. A complete clinical response was not observed. No patient developed distant metastases during the treatment. According to the response categories mentioned, $11(26 \%)$ patients were grouped as "responders" and 31 (74\%) as "nonresponders".

\section{Surgery results and surgical pathology}

All 42 patients became eligible for resection after the final staging; distant metastases did not evolve during the neoadjuvant treatment. In 32 of the 42 evaluable patients $(76 \%)$ a complete resection was achieved. In 10 patients, the resection was incomplete; however, in all of them total gastrectomy was performed. Seven of 10 patients with nonintestinal tumors had developed peritoneal spread during neoadjuvant chemotherapy at the time of surgery, 1 patient had a T4 carcinoma invading the pancreatic head, and 2 patients had retroperitoneal lymph node metastases distal to the renal veins. Postoperative morbidity was $19 \%(8 / 42)$. There was no 30 -day or in-hospital mortality. Median stay in the Intermediate Care Unit was 5 days (range, 1 to 7 days), and the median time to discharge was 15 days.
According to the postoperative pTNM classification, 4 patients were staged as ypT1 and 23 as ypT2. Twentyone of these 23 patients had tumors localized in the upper third of the stomach or in the region of the lesser curvature presenting with subserosal invasion. Ten patients were categorized as pT3 and 5 patients as pT4. Eleven patients had no lymph node metastasis (pN0); 2 of these patients were nonresponders and 9 were responders. The majority of the patients $(31 / 42 ; 74 \%)$ had lymph node involvement (pN1, 15 patients; $\mathrm{pN} 2$, 9 patients; $\mathrm{pN} 3,7$ patients).

A pathohistological complete response was not observed. Major pathological tumor regression was defined as fewer than $10 \%$ of vital tumor cells visible in the resected specimen, and this was reported in $7(17 \%)$ patients. Compared with pretherapeutic staging based on EUS, 30 patients had lower pT categories according to pathology. In 10 patients there was no change of the pT category and in 2 patients with uT3 tumors a pT4 category was reported.

\section{Survival and first site of failure}

The median duration of follow-up for the 16 surviving patients was 58 months (range, 38 to $80+$ months). Median survival of all 49 patients on an intent-to-treat analysis was 29 months (range, 5.8-80 months; Fig. 1). The median survival time for patients staged clinically as T3 $(n=32)$ was 32 months, while that for patients with cT4 tumors $(n=10)$ was 28 months (Fig. 2$)$. The median survival time for patients with pathohistological T1 and pT2 tumors $(n=27)$ was 32 months; for patients with pT3 and pT4 tumors $(n=15)$, it was 18 months 


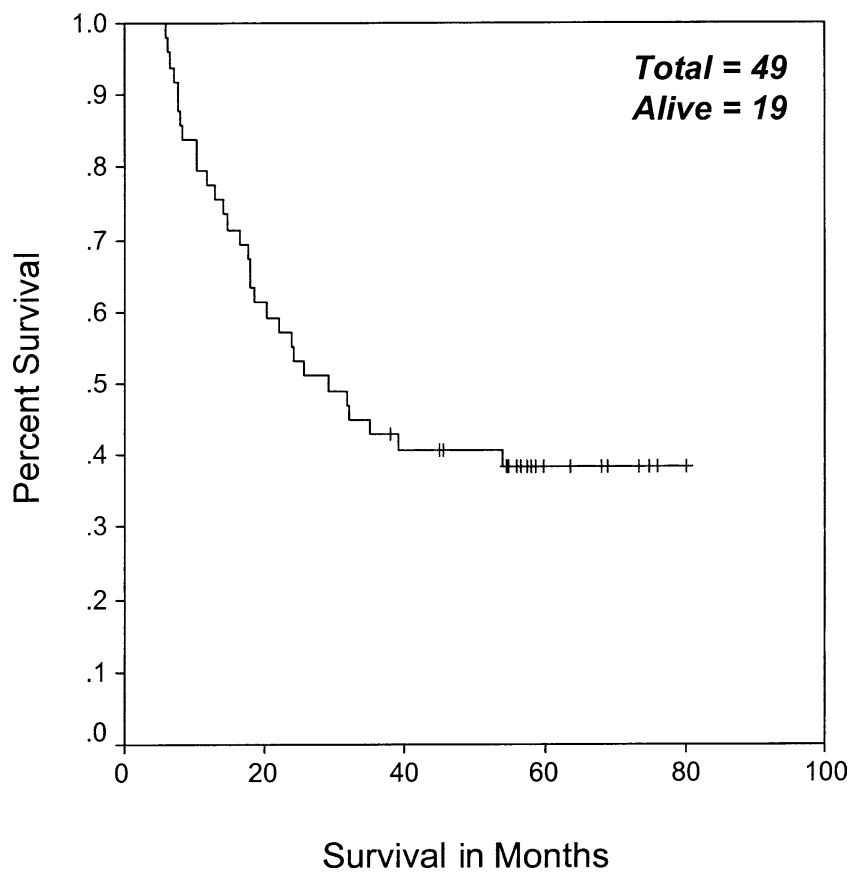

Fig. 1. Survival, calculated by the Kaplan-Meier method, of all 49 patients with locally advanced gastric cancer (uT3-4, $\mathrm{N}+, \mathrm{M} 0$ ) included in the study, on intent-to-treat analysis. Median survival was 29 months

(Fig. 3). The median survival time for the 42 patients assessable for response was 25.4 months (range, 6-80 months). After complete resection, the median survival time was 32 months (range, 7.6-80 months). After incomplete resection, the survival rate was 7.5 months (range, 5.8-73 months; $P<0.001$; Fig. 4). The median survival time for clinically responding patients has not yet been reached, but for clinically nonresponding patients it was 20 months (range, 6 to 80 months; $P<0.001$; Fig. 5).

The first site of failure was analyzed for all 32 completely resected patients. Twelve patients $(38 \%)$ are still alive with no evidence of disease. Of the 20 relapsed patients, 3 patients ( $9 \%$ ) are alive with known recurrences, and 17 patients (53\%) died of metastatic gastric cancer. The median time to recurrence was 19 months (range, 3.2 to 74 months). The first sites of failure are summarized in Table 3. The pattern of failure differed significantly according the Laurén classification: of 7 patients with intestinal-type tumors, 4 had lymph node recurrence, 2 had distant organ metastases, and 1 developed peritoneal carcinomatosis (PC). Nine of the 13 patients (69\%) with nonintestinal tumors had PC. Peritoneal spread in these 9 patients was combined with lymph node recurrence in 2 patients, with locoregional recurrence in another 2 patients, and with distant metastases also in 2 patients.

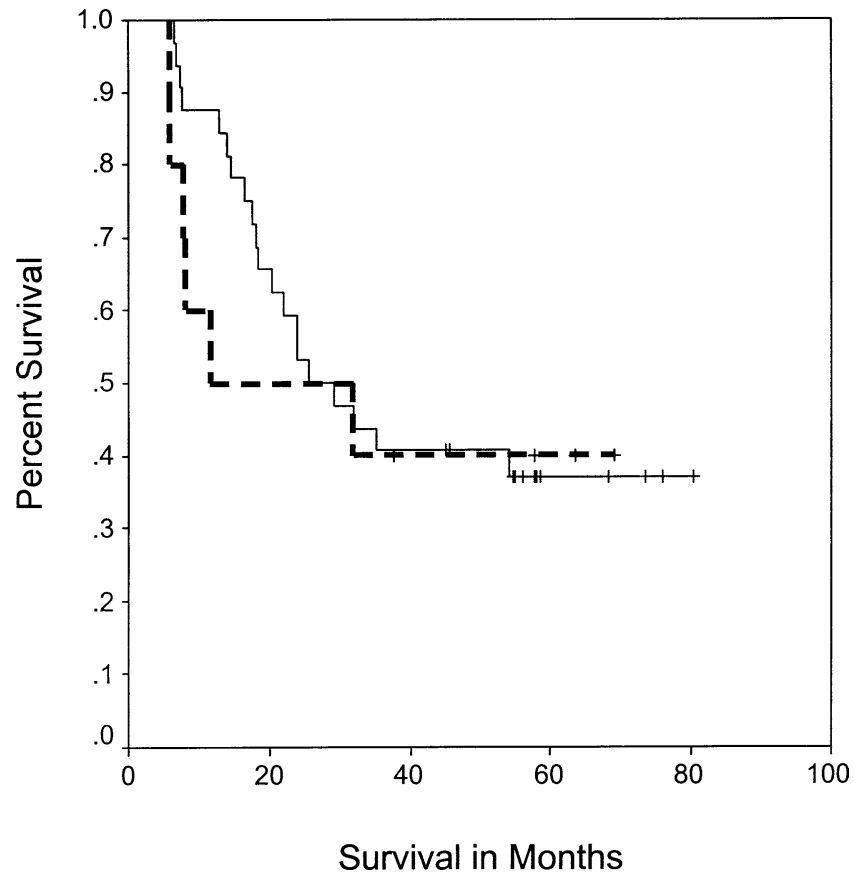

Fig. 2. Plot of overall actuarial survival (Kaplan-Meier) of the patients staged clinically as having T3 $(n=32$; continuous line) or T4 ( $n=10$; dashed line) tumors. Differences in survival were not significant

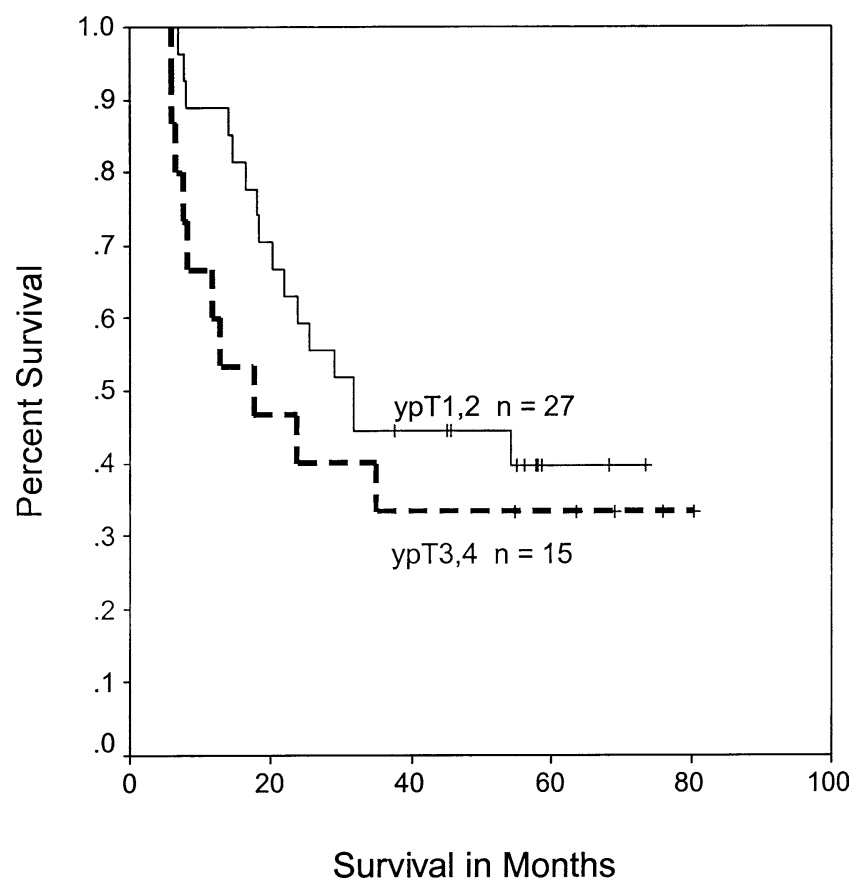

Fig. 3. Plot of overall actuarial survival (Kaplan-Meier) according to the pathohistological staging. There is no statistically significant difference between patients staged as ypT1 or ypT2 ( $n=27$; continuous line $)$ and patients staged as ypT3 or ypT4 $(n=15 ;$ dashed line $)$ 


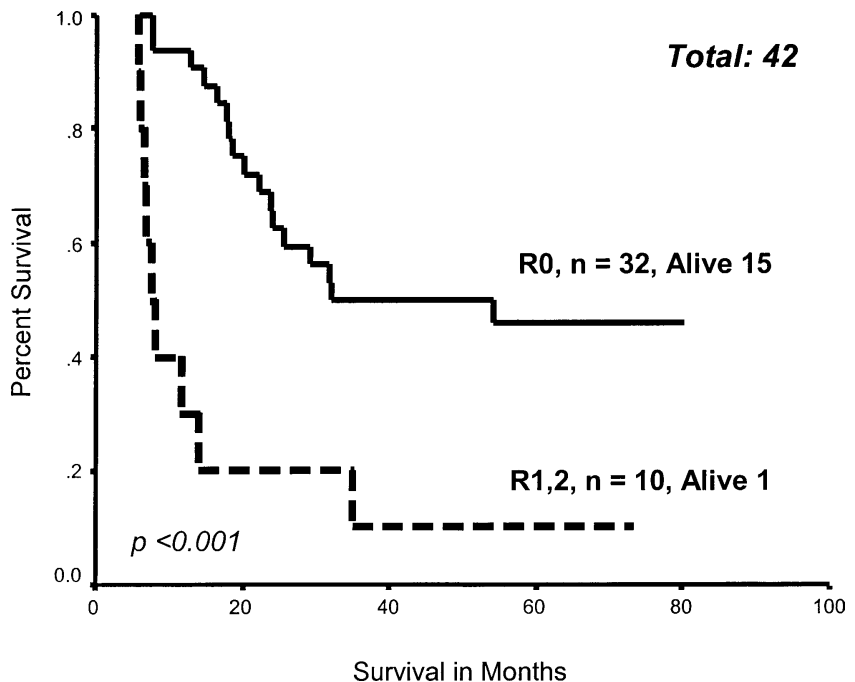

Fig. 4. Plot of overall actuarial survival of the 42 evaluable patients, according to the $\mathrm{R}$ category achieved, calculated by the Kaplan-Meier method

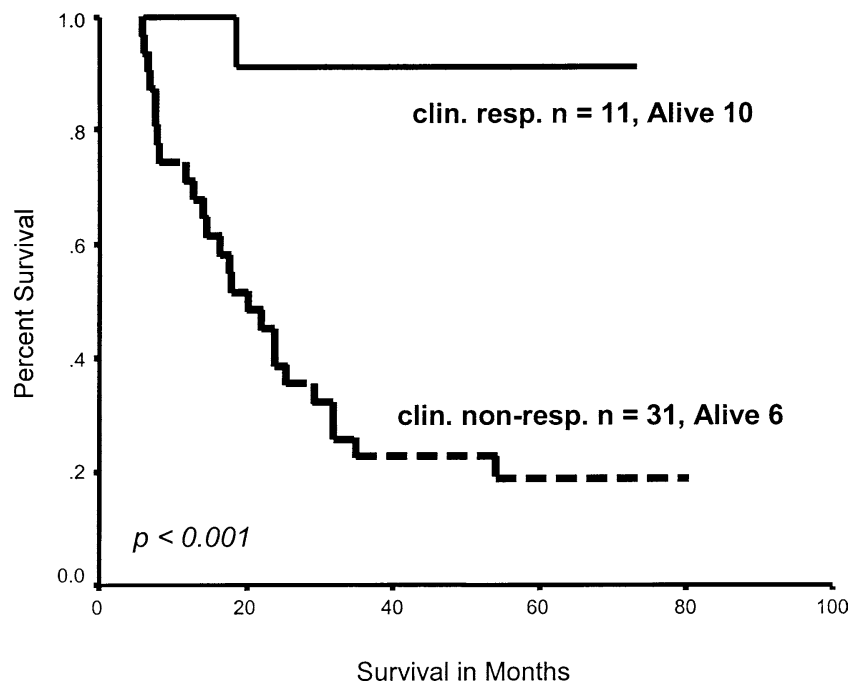

Fig. 5. Plot of overall actuarial survival of the 42 evaluable patients according to response category, calculated by the Kaplan-Meier method. clin. resp., clinical responders; clin. non-resp., clinical nonresponders

Table 3. Sites of first failure for evaluable and completely resected patients $(n=32)$;

20 of whom had recurrences

\begin{tabular}{lcccc}
\hline Site of failure & $\begin{array}{c}\text { Total } \\
\text { number }\end{array}$ & $n$ & $\begin{array}{c}\text { Nonintestinal } \\
\text { type }\end{array}$ & $\begin{array}{c}\text { Intestinal } \\
\text { type }\end{array}$ \\
\hline $\begin{array}{l}\text { Distant failure } \\
\text { - Liver }\end{array}$ & 3 & 2 & 1 & 2 \\
- Ovary & & 1 & 1 & 1 \\
Peritoneal failure & 10 & & 0 & 1 \\
- Alone & & 4 & 9 & 1 \\
- + Lymph nodes & & 2 & 3 & 1 \\
- +Local recurrence & & 2 & 2 & 0 \\
- +Distant (ov/cut) & & & 2 & 0 \\
Lymph node failure & 7 & 5 & 3 & 0 \\
- Alone & & 1 & 1 & 4 \\
- +Local recurrence & & 1 & 1 & 0 \\
- +Distant (oss) & & & 1 & 0 \\
\hline
\end{tabular}

ov/cut, ovary/cutaneous; oss, osseous

\section{Discussion}

Despite numerous phase II studies that have investigated neoadjuvant chemotherapy in locally advanced gastric cancer, the true impact of the additional preoperative treatment has not been finally established. The survival data after neoadjuvant chemotherapy and subsequent resection in the published phase II studies vary between 13 and 30 months (median) [7-9,11,12,18, $23,24]$. These differences in survival might result from a variety of factors, which include different staging modalities, different study designs, different chemotherapy regimens applied, and also different surgical resection strategies. So far, no conclusive results of phase III studies are available $[25,26]$.
Formerly, using the EAP (etoposide, adriamycin, and cisplatin) chemotherapy regimen, we and others observed favorable clinical responses in about $60 \%$ of patients (clinical complete and partial response) [27,28]. However, this was achieved only with a high toxicity, often requiring dose reductions and hospital admissions. The longterm results using the EAP regimen appear to be promising, but, due to the high toxicity, this regimen is unsuitable for a phase III trial. Therefore, effective and low toxic regimens, which can be given on an outpatient basis, have been developed. Biweekly cisplatin in combination with weekly high-dose folinic acid (HD-FA)/high-dose 5-fluorouracil (HD-5-FU) (PLF) has shown a high antitumor activity, of up to $70 \%$, with a reasonable toxicity profile, in patients with 
metastatic gastric cancer [16]. Based on these results we investigated the antitumor activity and toxicity of the PLF regimen, given in a neoadjuvant fashion, in patients with locally advanced gastric cancer.

The overall toxicity of the neoadjuvant PLF regimen was moderate, and, in particular hematologic toxicity was remarkably low. Thrombocytopenia grade 3 and 4 was not observed, leucopenia grade 3 was noted in 3 patients only, and anemia was moderate. A major concern was thromboembolic complications. In six patients, thrombosis of the subclavian vein occurred at the site of the port implantation. One patient developed pulmonary embolism after thrombosis of the femoral vein. These complications might be due to the known fact of an enhanced risk of thromboembolism in cancer patients; or they might be chemotherapy-related, because of possible hemostatic changes that occur during the HD-5-FU treatment [29,30]. However, after subsequent anticoagulation using low-dose heparin once daily for all patients, no further thrombotic complications were observed. Therefore, low-dose anticoagulation during neoadjuvant PLF treatment is strongly recommended [31].

In contrast to the high remission rates reported in other studies, the rate of $26 \%$ of clinically responding patients in our study appears to be low. Furthermore, we did not observe any complete pathohistological response $[18,27]$. The reasons for this may be our homogeneous high-risk patient population with uT3/uT4 primary tumors and the meticulous pathohistological workup of the resected specimen using antibodies against cytokeratin to detect even small residual nests of tumor cells. The rate of macroscopic and microscopic complete resection (UICC-R0) in this investigation was $76 \%$, which is comparable to that in other phase II trials $[18,23]$.

One pivotal question in this context is whether neoadjuvant treatment really can lead to the downstaging of a primary tumor. In our study, the number of patients found to have pT1 and pT2 primary tumors after resection was particularly high, similar to reports by other groups $[12,18]$. However, this high rate of downstaging might have been overestimated. The major subgroup of tumors contributing to "downstaging" are the tumors classified in pathology as ypT2. Using EUS in the staging of gastric cancer, problems especially arise in differentiating the $\mathrm{T} 2$ from the $\mathrm{T} 3$ category. In this respect, the sensitivity of EUS is only $64 \%$ [32]. Furthermore, in areas of the stomach with no serosal covering, especially in proximal gastric cancer and cancer in the lesser curvature, tumors do not reach the serosal lining and are consequently classified as pT2 by pathology $[33,34]$. Accordingly, the survival rates of patients with proximal pT2 tumors are comparable to those in patients with pT3 tumors in the middle and

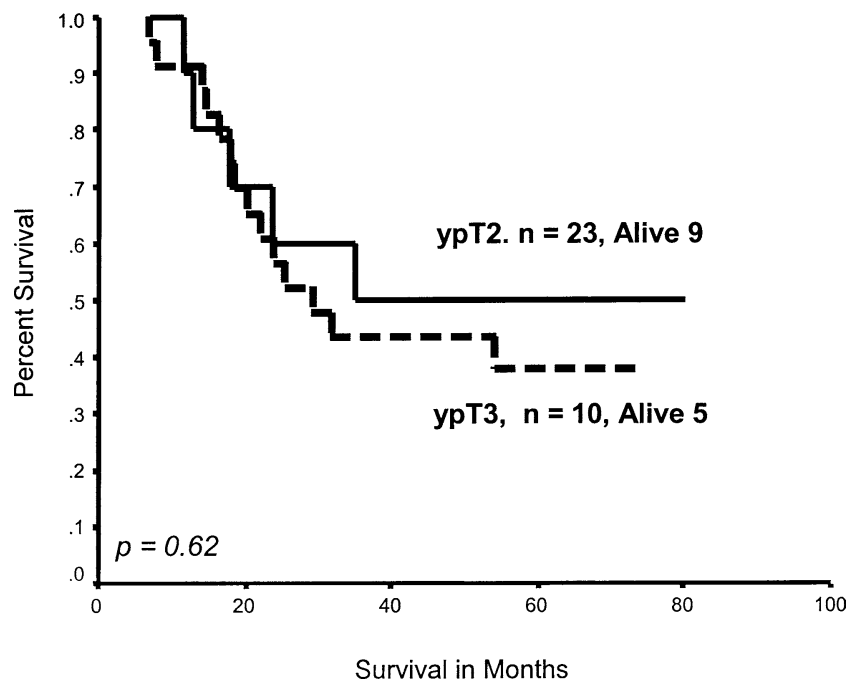

Fig. 6. Plot of overall actuarial survival of ypT2 vs ypT3 carcinomas, calculated by the Kaplan-Meier method. The two curves are nearly congruent

distal part of the stomach, an observation that was also apparent in this study (Fig. 6) [35]. Only in the sixth edition of the UICC guidelines for the staging of gastric cancer [36], valid as from January 2003, is there a differentiation between pt2a (tumor invades muscularis propria) and pT2b (tumor invades subserosa); however, this was not taken into account in the actual stage grouping system of the UICC.

In contrast to the subjective phenomenon of downstaging or downshrinking of the primary is the favorable complete resection rate of $76 \%$ in this study. This demonstrates a benefit for neoadjuvant chemotherapy. In the Pattern of Care Study of the American College of Surgeons [1] and in the German Gastric Carcinoma Study Group [2], an R0 resection rate of only about $40 \%$ was reported with surgery alone in locally advanced gastric cancer. Following complete resection, responding patients have an impressive survival advantage [37].

Following complete resection, distant metastases as first site of failure were rare. This may be due to the systemic effect of chemotherapy on clinically occult micrometastases [27]. Isolated local recurrence was also a rare event. This might be a result of the influence of the extended D2 lymph node dissection, which minimized the risk of local failure [38]. Lymph node failures occurred only in nonresected lymph node echelons. A major area of concern is the development of peritoneal dissemination (PC), not only during follow-up but also already during neoadjuvant treatment, despite inconspicuous findings on laparoscopy. At the time of surgery, 7 patients exhibited manifest PC; all of them were nonresponders to chemotherapy and had tumors of the nonintestinal subtype [39]. Despite complete resection, 
10 of $22(45 \%)$ relapsing patients developed PC alone or together with additional metastases during follow-up. This observation raises the question of whether intravenous chemotherapy is able to affect intraperitoneal dissemination. In a recent published multivariate analysis of longterm survivors of gastric cancer after complete resection, only the Laurén classification emerged as a prognostic factor, with nonintestinal type faring significantly less well [40]. In patients with the non-intestinal subtype, neoadjuvant chemotherapy, combining preoperative systemic and intraperitoneal chemotherapy, may be able to improve outcome [6,12].

In summary, this phase II study demonstrates that the PLF regimen has low toxicity and can be applied safely on an outpatients basis. The PLF regimen shows adequate response rates and a subsequent high rate of complete resection, with low postoperative morbidity and no postoperative mortality. Responding patients have an excellent prognosis. In nonintestinal type tumors, the development of peritoneal dissemination even during treatment - was not influenced by the intravenous neoadjuvant chemotherapy. An improvement of the results currently obtained seems to be possible by the introduction of additional therapeutic strategies, such as intraperitoneal chemotherapy given in a neoadjuvant or adjuvant fashion. Up to now, neoadjuvant therapy remains an experimental approach in resectable and locally advanced gastric cancer, and therefore should be performed only within innovative clinical trials.

\section{References}

1. Wanebo HJ, Kennedy BJ, Chmiel J, Steele G Jr, Winchester D, Osteen R. Cancer of the stomach. A patient care study by the American College of Surgeons. Ann Surg 1993;218:583-92.

2. Siewert JR, Böttcher K, Stein H, Roder JD, and the German Gastric Carcinoma Study Group. Relevant prognostic factors in gastric cancer. Ann Surg 1998;228:449-61.

3. Kelsen D. Adjuvant and neoadjuvant therapy for gastric cancer. Semin Oncol 1998;23:379-89.

4. Ajani JA, Mansfield PF, Ota DM. Potentially resectable gastric carcinoma: current approaches to staging and preoperative therapy. World J Surg 1995;19:216-20.

5. Lowy AM, Leach SD. Adjuvant/neoadjuvant chemoradiation for gastric and pancreatic cancer. Oncology (Huntingt) 1999;13:12130.

6. Leichman L, Silberman H, Leichman CG, Spears CP, Ray M, Muggia FM, et al. Preoperative systemic chemotherapy followed by adjuvant postoperative intraperitoneal therapy for gastric cancer: a University of Southern California pilot program. J Clin Oncol 1992;10:1933-42.

7. Ajani JA, Mayer RJ, Ota DM, Steele GD, Evans D, Roh M, et al. Preoperative and postoperative combination chemotherapy for potentially resectable gastric carcinoma. J Natl Cancer Inst 1993:85:1839-44.

8. Ajani JA, Roth JA, Putnam JB, Walsh G, Lynch PM, Roubein LD, et al. Feasibility of five courses of pre-operative chemo- therapy in patients with resectable adenocarcinoma of the oesophagus or gastrooesophageal junction. Eur J Cancer 1995;31A: 665-70.

9. Rougier P, Mahjoubi M, Lasser P, Ducreux M, Oliveira J, Ychou $\mathrm{M}$, et al. Neoadjuvant chemotherapy in locally advanced gastric carcinoma - a phase II trial with combined continuous intravenous 5-fluorouracil and bolus cisplatinum. Eur J Cancer 1994; 30A:1269-75.

10. Fink U, Stein HJ, Schuhmacher C, Wilke HJ. Neoadjuvant chemotherapy for gastric cancer: update. World J Surg 1995;19:50916.

11. Alexander HR, Grem JL, Hamilton JM, Pass HI, Hong M, Fraker DL, et al. Thymidilate synthase protein expression: association with response to neoadjuvant chemotherapy and resection for locally advanced gastric and esophageal adenocarcinoma. Cancer J 1995;1:49-54.

12. Kelsen D, Karpeh M, Schwartz G, Gerdes H, Lightdale C, Botet J, et al. Neoadjuvant therapy of high-risk gastric cancer: a phase II trail of preoperative FAMTX and postoperative intraperitoneal fluorouracil-cisplatin plus intravenous fluorouracil. J Clin Oncol 1996;14:1818-28.

13. Siewert JR, Sendler A. The current management of gastric cancer. Adv Surg 1999;33:69-93.

14. Kelsen D, Karpeh M, Schwartz G, Christman K. Neoadjuvant and postoperative chemotherapy for high risk patients with gastric cancer (abstract). Proc Am Soc Clin Oncol 1994;13:195.

15. Siewert JR, Fink U, Sendler A, Becker K, Böttcher K, Feldmann HJ, et al. Gastric cancer. Curr Probl Surg 1997;43:837-937.

16. Wilke H, Korn M, Köhne C, Fink U, Preusser P, Vanhoefer U, et al. Phase II results of weekly infusional high-dose 5-FU (HD-5FU) plus folinic acid (FA) and biweekly cisplatin (C) for advanced gastric cancer (abstract). Ann Oncol 1996;7 (Suppl 5):46.

17. Sendler A. Response evaluation - pathway for accurate indications development. Kongressbd Dtsch Ges Chir Kongr 2001;118: 169-71.

18. Ajani JA, Mansfield PF, Lynch PM, Pisters PW, Feig B, Dumas P, et al. Enhanced staging and all chemotherapy preoperatively in patients with potentially resectable gastric carcinoma. J Clin Oncol 1999;17:2403-11.

19. Japanese Research Society for Gastric Cancer. Japanese classification of gastric carcinoma. Tokyo: Kanehara; 1995. 1: pp. 1-35.

20. UICC. TNM classification of malignant tumors. 4th Ed. Berlin Heidelberg, New York: Springer-Verlag; 1997.

21. American Joint Committee on Cancer. Manual for staging of cancer. 2nd. Ed. Philadelphia: JB Lippincott; 2000.

22. Becker K, Mueller J, Fink U, Busch R, Siewert JR, Höfler H. Morphologische Responseevaluierung beim neoadjuvant chemotherapierten Magenkarzinom. Verh Dtsch Ges Pathol 2000;84: 164-74.

23. Fink U, Schuhmacher C, Stein HJ, Busch R, Feussner H, Dittler $\mathrm{HJ}$, et al. Preoperative chemotherapy for stage III-IV gastric carcinoma: feasibility, response and outcome after complete resection. Br J Surg 1995;82:1248-52.

24. Crookes P, Leichman CG, Leichman L, Tan M, Laine L, Stain S, et al. Systemic chemotherapy for gastric carcinoma followed by postoperative intraperitoneal therapy: a final report. Cancer 1997; 79:1767-75.

25. Songun I, Keizer HJ, Hermans J, Klementschitsch P, de Vries JE, Wils JA, et al. Chemotherapy for operable gastric cancer: results of the Dutch randomised FAMTX trial. The Dutch Gastric Cancer Group (DGCG). Eur J Cancer 1999;35:558-62.

26. Kang YK, Choi DW, Im YH, Kim CM, Lee JI, Moon NM, et al. A phase III randomized comparison of neoadjuvant chemotherapy followed by surgery versus surgery for locally advanced stomach cancer (abstract). Proc Am Soc Clin Oncol 1996;15:215.

27. Wilke H, Preusser P, Fink U, Gunzer U, Meyer HJ, Meyer J, et al. Preoperative chemotherapy in locally advanced and nonresectable gastric cancer: a phase II study with etoposide, doxorubicin, and cisplatin. J Clin Oncol 1989;7:1318-26. 
28. Schuhmacher CP, Fink U, Becker K, Busch R, Dittler HJ, Mueller $\mathrm{J}$, et al. Neoadjuvant therapy for patients with locally advanced gastric carcinoma with etoposide, doxorubicin, and cisplatinum. Closing results after 5 years of follow-up. Cancer 2001;91:918-27.

29. Poorter RL, Lauw FN, Bemelman WA, Bakker PJ, Taat CW, Veenhof $\mathrm{CH}$. Complications of an implantable venous access device (Port-a-Cath) during intermittent continuous infusion of chemotherapy. Eur J Cancer 1996;32A:2262-6.

30. Loreto MF, De Martinis M, Corsi MP, Modesti M, Ginaldi L. Coagulation and cancer: implications for diagnosis and management. Pathol Oncol Res 2000;6:301-12.

31. Tesselar MET, Ouwerkerk J, Rosendaal FR, Osanto S. Prophylaxis with low molecular weight heparin reduces the risk for catheter-related venous thrombosis in cancer patients in centrally, but not in peripherally inserted central venous catheters (abstract). Proc Am Soc Clin Oncol 2001;19:1584.

32. Rösch TH. Endosonographic staging of gastric cancer: a review of literature results. Gastrointest Clin North Am 1995;3:549-57.

33. Siewert JR, Böttcher K, Bollschweiler E. Die TNM Klassifikation beim Magenkarzinom - das Problem der T2 Tumore. Dtsch Med Wochenschr 1991;116:473-5.

34. Hundahl SA, Phillips JL, Menck HR. The National Cancer Data Base Report on poor survival of U.S. gastric carcinoma patients treated with gastrectomy: fifth edition American Joint Committee on Cancer staging, proximal disease, and the "different disease" hypothesis. Cancer 2000;88:921-32.

35. Siewert JR, Böttcher K, Stein HJ, Roder JD, Busch R. Problem of proximal third gastric carcinoma. World J Surg 1995;19:52331.

36. UICC. TNM Classification of malignant tumours. 6th Ed. Berlin Tokyo Heidelberg New York: Springer-Verlag; 2002.

37. Macdonald JS, Smalley SR, Benedetti J, Hundahl SA, Estes NC, Stemmermann GN, et al. Chemoradiotherapy after surgery compared with surgery alone for adenocarcinoma of the stomach or gastroesophageal junction. N Engl J Med 2001;345:72530 .

38. Yoo CH, Noh SH, Shin DW, Choi SH, Min JS. Recurrence following curative resection for gastric carcinoma. Br J Surg 2000;87: 236-42.

39. Weiss M, Eder M, Bassermann R. Charakterisierung verschiedener Magenkarzinomtypen mit unterschiedlicher Metastasierung in Leber, Peritoneum und Knochen. Pathologe 1993; 14:260-4.

40. Hochwald SN, Kim S, Klimstra DS, Brennan MF, Karpeh MS. Analysis of 154 actual 5-year survivors of gastric cancer. J Gastrointest Surg 2000;4:520-5. 